\title{
Repetitive speech disorder resulting from infarcts in the paramedian thalami and midbrain
}

\author{
Kazuo Abe, Ritsuko Yokoyama, Shiro Yorifuji
}

\begin{abstract}
A repetitive speech disorder resulting from infarcts in the paramedian thalami and the midbrain is reported. Although the speech disorder seemed like stuttering, the compulsive repetitions, constant rate and monotonous tone were not associated with ordinary stuttering. Since repetition was restricted to the first syllable, the speech disorder in our patient could be distinguished from palilalia. The extrapyramidal system is considered responsible for repetitive speech disorders resulting from infarcts in the paramedian thalami and the midbrain but without good reason. Repetitive speech disorder in patients with infarcts in the supplementary motor area (SMA) have similar clinical features to our patient. It is suggested that interruption in the projective system to the SMA is a possible cause of "stuttering like repetition".
\end{abstract}

(F Neurol Neurosurg Psychiatry 1993;56:1024-1026)

Stuttering is characterised by difficulty in producing the next expected sound. ${ }^{1-3}$ Although congenital stuttering is well known, acquired stuttering resulting from cerebrovascular disease also occurs. Various types of cerebral infarcts can cause acquired stuttering, but stuttering resulting from infarcts in the paramedian thalami and paramedian midbrain has not been previously reported. We report the first case, and discuss its unique clinical features compared with those of other speech disorders resulting from infarcts in the same anatomical location.

\section{Case report}

A 38 year old man had been suffering from cardiovascular disease. He was admitted to a

Table Frequency of stuttering is evaluated in four modalities; repetition of spoken phrase, reading phrases, naming, and spontaneous speech. Stuttering is the most frequently observed in spontaneous speech.

\begin{tabular}{|c|c|c|c|c|c|c|c|}
\hline Category & Frequency & Rare if any & Seldom & Sometime & $\begin{array}{l}\text { Every } \\
\text { sentence }\end{array}$ & $\begin{array}{l}\text { Every two } \\
\text { syllables }\end{array}$ & $\begin{array}{l}\text { Every } \\
\text { syllable }\end{array}$ \\
\hline repeat & $5 \%(1 / 22)$ & & & & & & \\
\hline reading & $9 \%(2 / 22)$ & & & & & & \\
\hline naming & $28 \%(8 / 28)$ & & & & & & \\
\hline speech & $58 \%(14 / 24)$ & & & & & & \\
\hline
\end{tabular}

hospital and had coronary angiography. After the angiography, he was comatose and developed a tetraparesis. He had dilated non-reactive pupils. Doll's head movements were preserved. A CT scan 24 hours later showed low density areas in the paramedian thalami and in the midbrain. Three weeks later, he started to respond with simple words to verbal commands. However, he was still drowsy and seemed apathetic. Deep tendon reflexes were slightly increased and Babinski's sign was noted on both sides. Two months later, he began to speak in long sentences but showed compulsive repetition of syllables, almost always the first syllable, and never words or phrases. Neurological examination at this stage revealed that he was still apathetic and lay on the bed if other instructions were not given. He had isocoric pupils and preserved light reaction. He had disturbed eye movements on right lateral gaze and vertical gaze. He had preserved doll's head movements and Bell's phenomenon. Other normal cranial nerve functions were found. $\mathrm{He}$ had slightly increased deep tendon reflexes and positive Babinski's sign on both sides, and normal muscle strength with mild rigidity in the hand joints. There was increased jaw jerk and positive sucking reflex. Coordinative movements were normal. The patient could walk a short distance unaided, but with fluctuating and compulsive gate. Sensory functions were almost normal.

Repetition in his speech was restricted to syllables; repetition of words or phrases was not observed. Repetition of syllables was exclusively observed in spontaneous speech or in reply to a question and was rarely observed when he repeated words spoken to him, or when he read sentences (table). There were no other speech disorders, such as perseveration, literal or verbal paraphasia. For example, when asked what his breakfast was, his answer was "go-go-go-go-go-gogohan to misoshiru soreni ninn-ninn-ninnninn-ni-ni-ni-ni-ni-nishimetayatsu" (gohan = rice, to $=$ and , soreni $=$ and, misoshiru $=$ miso soup, nishimetayatsu = boiling foods).

Mild disorientation to place and time was noticed. Digit span was 6 forward and 3 backward. Recent verbal and visual memory were disturbed. Calculation and writing were also disturbed. Right left disorientation, apraxia and agnosia were absent. 
Figure MRI (1.5 Tesla super conducting system, spin-echo method,

$T R / T E=2500 / 120 \mathrm{~ms}$ ).

High intensity lesions are demonstrated in the paramedian thalami (right) and medial midbrain (left). The centromedian nucleus of the thalamus is involved. There are abnormal signals in the frontal white matter and in the white matter adjacent to the left trigone.

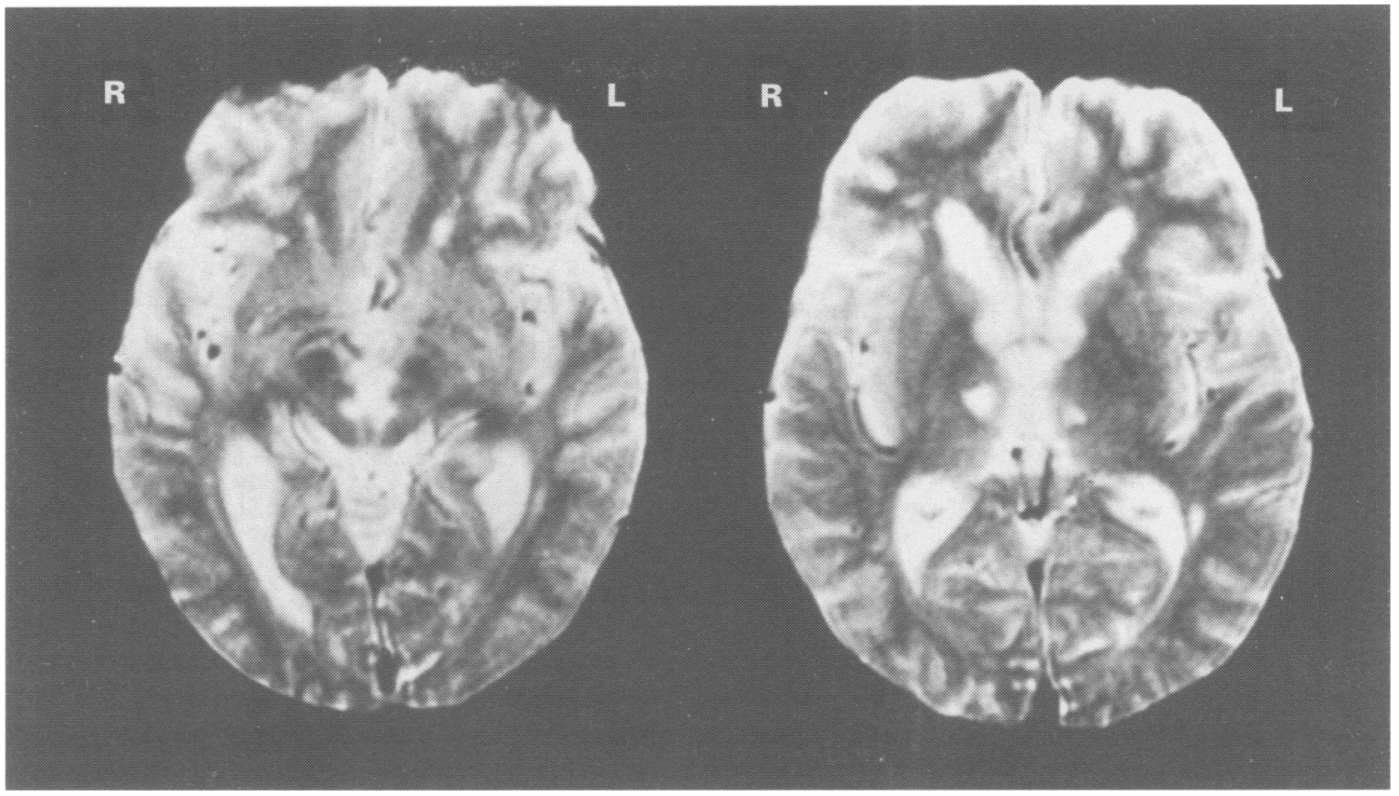

Laboratory findings including complete blood count and serum calcium, blood urea nitrogen, creatine phosphokinase, alkaline phosphatase, and glutamic transaminase were normal. An ECG was normal.

The basic rhythm on an EEG was $8 \mathrm{~Hz}$ alpha waves with complete alpha attenuation, and sporadic 5-6 $\mathrm{Hz}$ theta waves were seen bilaterally. A brainstem auditory evoked potential (BAEP) showed no abnormal findings. MRI showed infarcts in the bilateral medial thalami and in the midbrain (fig).

The repetitive speech disorder has continued for more than two years, although his daily activity gradually improved. Instability in walking was slightly improved, but there was no improvement in right eye movement.

\section{Discussion}

Stuttering can be defined as a deviation of speech attracting attention of speakers or listeners because of interruption of the normal rhythm of speech by involuntary repetition, prolongation or arrest of sounds. Acquired stuttering, mainly resulting from cerebral infarcts, has been described in disorders in the corpus striatum, the dominant temporal lobe, the dominant parietal lobe, or in the dominant operculas. ${ }^{1-3}$ Various types of speech disorders resulting from infarcts in the paramedian thalami and the midbrain have been reported, but stuttering has not been described. Castaigne ${ }^{4}$ studied patients with paramedian thalamic and midbrain infarcts and described some patients with mutism. He did not, however, report patients with repetitive disorders. Yasuda ${ }^{56}$ reported a patient with repetitive speech disorder resulting from infarcts in the paramedian thalami and midbrain and they diagnosed it as palilalia. The repetition shown by their patient was not restricted to the first syllables and showed gradually increasing rate with reduced loudness. In contrast, our patient showed repeti- tions restricted to the first syllables. Restricted repetition to the first syllable is a characterised feature of stuttering. ${ }^{5}$ Our patient did not demonstrate an increasing rate with reduced loudness, which is known as "palilalia aphone". Instead, he repeated the first syllables at a constant rate and loudness. His repetitive speech can therefore be distinguished from palilalia or logoclonia.

The repetitive speech disorder in our patient was unique for its extraordinary frequency of repetitions. He repeated the first syllables of words no less than seven times at a slow and constant rate, which was unlike stuttering. Conversely, palilalia resulting from infarcts in the paramedian thalami and midbrain showed repetitions of syllables or parts of words more frequently than repetitions of words or phases, which does not fit the criteria for palilalia. ${ }^{25-8}$ Since repetitive speech disorders resulting from infarcts in the paramedian thalami and midbrain have characteristic clinical features, it is natural to distinguish them from stuttering and palilalia. We therefore propose to call this repetitive speech disorder "stuttering like repetition".

Although the pathogenesis of "stuttering like repetition" is unclear, it seems that" it results from extrapyramidal dysfunction ${ }^{26}$ as in palilalia. "Stuttering like repetition", however, has clinical features which differ from those of palilalia, and our patient did not have extrapyramidal signs. We consider that the causative lesion for "stuttering like repetition" can not be restricted to the extrapyramidal system. Nagafuchi reported a patient with stuttering resulting from infarcts in the supplementary motor area (SMA), and the clinical features of their patient were compatible with ours. ${ }^{9}$ As some patients with infarcts in the SMA demonstrated uncontrollable might play a significant role both in initiation and in control of spontaneous speech. ${ }^{10-12}$ The infarcts of our patient anatomically interruption of speech or utterance, the SMA 
located in the centromedial thalami and to the cerebral peduncles which are part of the projection system to the SMA. ${ }^{13}$ We concluded that interruption of the projective system to the SMA might cause "stuttering like repetition".

1 Helm NA, Butler RB, Benson DF. Acquired stuttering. Neurology (NY) 1978;28:1156-65.

2 Ardila A, Lopez MV: Progressive stuttering associated with right hemisphere lesion. Brain Lang 1986;27: 239-46.

3 Cliporrotti L, Bisichi PS, Denes G. Acquired stuttering: a motor programming disorder? Eur Neurol 1988;28: motor

4 Castagine P, Lhermitte F, Buge A, et al. Paramedian thalamic and midbrain infarcts; Clinical and neuropathological study. Ann Neurol 1981;10:127-48.

5 Yasuda Y, Akiguchi I, Ino M, et al. Paramedian thalamic and midbrain infarcts associated with palilalia. $\mathcal{F}$ Neurol Neurosurg Psychiatry 1990;53:797-9.

6 Hasegawa O, Kobayashi H, Yoshii Y. A case of palilalia with paramedian thalamic and midbrain infarcts. with paramedian thalamic and midbrain

7 Marie P, Levy G. Un sigulier trouble de la parole. Monde Med 1925;35:6-80.

8 Nakura H, Sugishita M, Nizuma H. A case of aphasia associated with palilalia. Neurological Medicine (Tokyo) 1982;16:554-8

9 Nagafuchi $M$, Takahashi T. Speech disorders due to damage of the left premotor area. Onseigengogaku 1989; 30:328-33.

10 Bhatnagar SC, Jandy O. Alleviation of acquired stuttering with human centromedian thalamic stimulation. Neurol Neurosurg Psychiatry 1989;52:1182-4.

11 Jonas $\mathrm{S}$. The supplementary motor region and speech emission. f Comm Disord 1981;14:349-73.

12 Penfield $W$, Welch $K$. The supplementary motor area of the cerebral cortex. A clinical and experimental study. the cerebral cortex. A clinical and experimen
Arch Neurol Psychiat (Chic) 1951;66:289-317.

13 Brodal A. Neurological anatomy in relation to clinical medicine. Oxford: Oxford University Press, 1981: medicine. 